\title{
Introduction to Multidimensional Scaling and Its Applications
}

\author{
Mark L. Davison

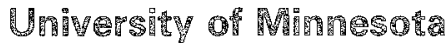

Although Richardson (1938) and Young and Householder (1938) may have officially initiated the multidimensional scaling (MDS) literature in psychology, frequent applications did not begin to appear until the seminal papers on nonmetric MDS by Shepard (1962) and Kruskal (1964). Twenty years later, it is time to critically examine the MDS literature and its contribution to psychology. The frrst two papers in this special issue review statisical developments in MDS with an emphasis on the design of MDS studies. The last four papers scrutinize the MDS research in four areas of common application: consumer, social, cognitive, and vocational psychology.

Carroll and Arabie (1980) have described two ways to define MDS. According to the broader of the two definitions, MDS means a set of techniques for estimating parameters in geometric models so as to yield a representation of data structure. Such a broad definition would encompass cluster, discriminant, and factor analysis. These techniques are treated here as altematives to MDS, rather than as methods included within it. In this special issue, the MDS literature refers to a body of knowledge involving (1) a set of statistical techniques for estimating the parameters in and assessing the fit of various spatial distance models for proximity or

APPLIED PSYCHOLOGICAL MEASUREMENT Vol. 7, No. 4, Fall 1983, pp. 373-379

(C) Copyright 1983 Applied Psychological Measurement Inc. 0146-6216/83/040373-07\$1.60 preference data and (2) the coordinate representations of stimulus structure that result from such statistical techniques.

This introduction first briefly reviews the past 50 years of developments in MDS, developments covered more extensively by Coxon (1982), Davison (1983), Kruskal and Wish (1978), and Schiffman, Reynolds, and Young (1981). Then it summarizes the six papers that follow.

\section{Development of MDS}

MDS techniques differ on several criteria. The more common models apply to symmetric proximity data, measurements defined over pairs of obm jects that quantify the degree to which the two objects are alike. Correlation coefficients and joint probabilities are two examples of proximity data. A proximity measurement may be called a measure of similarity or dissimilarity depending on whether the highest scores correspond to pairs that are most or least alike.

In contrast to models for symmetric proximity data are models for preference data. The data constitute measurements defined over pairs consisting of a stimulus and a subject. Each measurement quantifies a subject's atraction to or liking for the stimulus object. This section will begin by briefly highlighting the development of models for proximity dala; then it will consider preference models. 


\section{Proxinnity Models}

Proximity data can be classified as direct or derived. To obtain direct proximity data, the experimenter asks the subject to respond to stimulus pairs on the basis of similarity. Subjects might rate or rank pairs in terms of similarity. Many kinds of measures do not constitute proximity measurements, but they do provide a basis for deriving such measures. For instance, ability test scores do not constitute proximity measures, but such scores can be used to compute correlations between pairs of tests, and hence to derive proximities. When the proximities data are computed from other measurements, they are called derived, rather than direct, proximities.

The statistical models for proximity data can be classified as two- or three-way depending on whether the data matrix has two or three dimensions. Twoway models for symmetric proximities presume a matrix in which rows and columns correspond to stimuli. Three-way models for symmetric data presume a matrix with two dimensions corresponding to stimuli and a third that corresponds to replications. Although the replications may be occasions or settings, they are usually subjects, and henceforth this paper will refer to replications as subjects. Whereas two-way models contain only stimulus parameters, three-way models contain parameters for both stimuli and subjects.

MDS models consist of at least two components. The first component, what Spence (this issue) calls a spatial function, is a combination rule for stimulus coordinates. In most MDS models for proximity data, the spatial function is some form of Minkowski distance function:

$d_{i j}=\left[\Sigma_{k}\left|x_{i k}-x_{j k}\right|\right]^{1 / p}$.

Hence, Heiser and Meulman (this issue) refer to a distance function rather than a spatial function. In Equation 1, $d_{i j}$ refers to the theoretical distance between stimuli $i$ and $j, x_{i k}$ and $x_{j k}$ refer to coordinates for stimuli $i$ and $j$ along dimension $k$, and $p$ is an exponent $(\geqslant 1)$ specified by the researcher. Although the Euclidean distance function in which $p=2$ is the most common, the literature also contains discussions of the city-block metric $(p=1)$ and the dominance metric $(p=\infty)$ (Arnold, 1971;
Attmeave, 1950). The second component is a data function, $f$, which maps the theoretical distance onto the observed data, $\delta_{i j}$, indexing the proximity of stimuli $i$ and $j .{ }^{1}$ Thus, most MDS models assume that the data have the form $\delta_{i j}=f\left(d_{i j}\right)$, where $d_{i j}$ either has the form shown in Equation 1, if the data have two ways, or some variant including subject parameters, if the data have three ways.

To a large extent, tracing the development of MDS algorithms means tracing alterations in assumptions about the spatial or data function. Each alteration has been accompanied by corresponding changes in the numerical analytic techniques needed to solve for parameters and by changes in the kinds of data to which MDS is applicable. MDS began with the simple model of Young and Householder (1938), which was subsequently elaborated by Torgerson (1958) and Gower (1966).

The metric two-way model. Torgerson (1952, 1958) presented the best known (to psychologists) development of the metric two-way model:

$\delta_{i j}=d_{i j}=\left[\Sigma_{k}\left(x_{i k}-x_{j k}\right)^{2}\right]^{1 / 2}$.

The model is simple in the sense that it contains no explicit error function, the data function is a strict equality, it contains no subject parameters, and the Minkowski exponent $p$ must equal 2. It was later expanded to handle data of the form $\delta_{i j}=d_{i j}+c$. Torgerson (1958) used the data $\delta_{i j}$ to estimate scalar products of the form

$\delta_{i j}^{*}=\Sigma_{k} x_{i k} x_{j k}$.

He then proposed using an Eckart-Young (1936) decomposition of the square symmetric scalar products matrix to estimate stimulus coordinates that were uniquely determined up to a rotation.

Because of its restrictive assumptions, Torgerson's (1952) approach is seldom used today. It remains important, however, because more recent algorithms incorporate many of its features. Spence (this issue) concludes that when the data are lin-

\footnotetext{
'Authors in this special issue use different terms for the data and spatial functions. Spence calls the data function a distance function, which seems regrettable because the spatial function is often a Minkowski distance function. Heiser and Meulman break the data function into two subcomponents, fit and transformation functions.
} 
early related to distances, the Torgerson approach will often do as well as the nonmetric programs and do it more inexpensively.

Nonmetric two-way models. The early advances in nonmetric algorithms occurred from 1962 to 1968 (Guttman, 1968; Kruskal, 1964; Shepard, 1962; Young \& Torgerson, 1967). The major change was in the data function. In the less frequently used parametric mapping (Shepard \& Carroll, 1966), $f$ is considered to be a polynomial function specified by the user. In the more conventional monmetric models, $f$ is an unknown monotone function estimated by the algorithm. Whereas Torgerson's approach required interval or ratio level data, the nonmetric algorithms required only ordinal data.

Most recent algorithms allow sublist splitting in which the monotone function $f$ is allowed to vary for each row of the data matrix. Allowing $f$ to vary opened the way for conditional rank-order judgment tasks. In such tasks, one stimulus serves as a standard. The subject then ranks the $(n-1)$ remaining stimuli in terms of their similarity to the standard. Each stimulus in turn serves as a standard. The data element $\delta_{i j}$ represents the rank similarity of stimulus $j$ to standard $i$. Since the judgments in each row correspond to a different standard, the monotone function $f$ relating data to distances cannot be assumed constant across rows of the data matrix.

Not only did the nonmetric advances change assumptions about $f$, it allowed changes of assumptions about the Minkowski exponent, $p$, in the coordinate function. Most nonmetric algorithms allow the user to set $p$ at any desired value. Nevertheless, virtually all algorithms are computationally more efficient and dependable when $p=2$ for the Euclidean distance function (e.g., Kruskal, Young, \& Seery, 1973, p. 10). Computer programs that execute nonmetric analyses include MDSCAL (Kruskal \& Carmone, undated); KYST (Kruskal et al., 1973); TORSCA (Young \& Torgerson, 1967); ALSCAL (Young \& Lewyckyj, 1979); and MINISSA (Lingoes, 1973). Although these nonmetric models relaxed some assumptions of Torgerson (1952), they are still simple in two respects. They contain no subject parameters and no explicit assumptions about error.
Three-way models. In 1968 three-way models began to appear. The weighted Euclidean model (Bloxom, 1968; Carroll \& Chang, 1970; Horan, 1969), the most common-three way model, assumes subjects to vary in the importance or salience of the stimulus dimensions. Except for error, data are assumed to be of the form

$\delta_{i j s}=d_{i j s}=\left[\sum_{k} w_{k s}^{2}\left(x_{i k}-x_{j k}\right)^{2}\right]^{1 / 2}$

where

$\delta_{i j s}$ is the proximity measure on stimulus pair $(i, j)$ for subject $s$,

$d_{i j s}$ is the theoretical distance between $i$ and $j$ in a coordinate space unique to subject $s$,

$w_{k s}$ is a salience weight for subject $s$ along dimension $k$, and

$x_{i k}$ and $x_{j k}$ are stimulus coordinates as before.

Algorithms based on this model yield a dimensional description of stimuli, the coordinates $x_{i k}$, and a dimensional description of subjects, the weights $w_{k s}$. Both Carroll and Chang (1972) and Tucker (1972) have proposed more complex three-way models that allow subjects to vary both in their weightings and in their orientations of dimensions.

Programs for fitting the weighted Euclidean model include INDSCAL (Carroll \& Chang, 1970); SINDSCAL (Pruzansky, 1975); ALSCAL (Young \& Lewyckyj, 1979); and MULTISCALE (Ramsay, 1978). ALSCAL can fit a nonmetric form of the model in Equation 4; hence, it combines nonmetric and individual differences features.

Maximum likelihood models. Ramsay (1969) explicitly incorporated error assumptions into his models. In the log normal form of his metric twoway model, Ramsay assumed that each data point $\delta_{i j}$ is normally distributed about its expectation, $d_{i j}=\left[\sum_{k}\left(x_{i k}-x_{j k}\right)^{2}\right]^{1 / 2}$. Ramsay also has developed a $\log$ normal model that assumes that $\ln _{i j}$ is normally distributed about its expectation $d_{i j}$. Explicitly adopting assumptions about error allowed Ramsay to develop maximum likelihood estimates of coordinates and a $\chi^{2}$ statistic for testing the fit of the model to data. Although the $\chi^{2}$ statistic must be interpreted cautiously (Ramsay, 1980), it opens the way to a more confirmatory, less exploratory 
approach to MDS. Heiser and Meulman (this issue) discuss a more confirmatory approach to MDS.

Ramsay (1978) has subsequently developed maximum likelihood approaches to fiting the weighted Euclidean model in Equation 4, as well as approaches that allow the data function to be a polynomial function specified by the user. Takane (1981; Takane \& Sergent, 1983) has extended the maximum likelihood approach. Ramsay's (1978) MULTISCALE is the major program to implement the maximum likelihood approach.

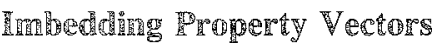

Not wanting to rely solely on subjective visual inspection of coordinates for interpretation of solutions, researchers have used various statistical techniques to confirm (or disconfirm) their interpretations of coordinates. If the coordinates, $x_{i k}$, correspond to locations along stimulus attributes (e.g., price, size, status, leadership potential), then it would be expected that a high correlation would be obrained by regressing an independent measure of the atribute onto the stimulus coordinates. Since the attributes can be considered stimulus properties, the process of regressing independent measures of attributes onto stimulus coordinates is sometimes called imbedding property vectors into the space. If the researcher has a prior hypothesis that a particular attribute will appear as a dimension in the MDS solution, then he or she can obtain independent measurements on the attribute. The multiple correlation obtained by regressing the independent measurements onto the MDS stimulus coordinates can be used to confirm or disconfirm the hypothesis. Davison (1983) and Kruskal and Wish (1978) have described in more detail the process of imbedding property vectors to interpret MDS solutions.

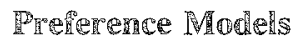

Whereas proximity data index the congruence between two stimuli, a preference datum, $\delta_{i s}$, indexes the degree to which subject $s$ likes or is attracted to stimulus $i$. As a rule, the data matrix has two dimensions corresponding to stimuli and subjects. Virtually all models contain parameters for both stimuli and subjects. The spatial function is not necessarily a distance function.

Carroll (1972) divided MDS of preference data into intemal and extemal analyses. In an intemal analysis, both stimulus and subject parameters are unknown and must be estimated. In an external analysis the stimulus coordinates are known and only subject parameters need be estimated. External methods are not stimulus scaling techniques, but they do yield subject measurements.

Models for internal analysis. Distance or unfolding models (Coombs, 1964) generally take the following form, except for error:

$\delta_{i s}=f_{s}\left(d_{i s}\right)$

$d_{i s}=\left[\sum_{k}\left(\mathrm{x}_{i k}-\mathrm{y}_{s k}\right)^{p} \rrbracket^{1 / p}\right.$

where

$y_{s k}$ is the coordinate locating subject $s$ along dimension $k$,

$d_{i s}$ is the theoretical distance between stimulus $i$ and the ideal point for subject $s$, and

$f_{s}$ is a function unique to subject $s$.

According to this model, the more a stimulus resembles the subject's ideal, the more the subject will like the stimulus.

Internal analyses based on distance functions have suffered from computational problems that limit their use. The nature of the problem differs somewhat depending on the form of $f_{s}$. If $f_{s}$ is considered to be an unknown monotone function, the solutions are prone to a form of degeneracy in which stimuli cluster in one region of the space and subjects cluster in another yielding an uninterpretable solution consisting of two clumps. Schönemann (1970) describes computational problems arising when $f_{s}$ is an equality. Kruskal, Young, and Seery (1973) have described the problem for an unknown monotone function $f_{s}$.

Algorithms based on linear, rather than distance, spatial functions have been more successful (Carroll, 1972; Tucker, 1960). Typically, such algorithms assume data of the following form except for error:

$\delta_{i s}=f_{s}\left[\sum_{k} w_{k s} x_{i k}\right]$.

where $w_{k s}$ is a salience weight for subject $s$ allong 
dimension $k$. Instead of assuming that there exists an optimum, $y_{s k}$, along each dimension, and that stimuli are preferred to the extent they resemble the optimum (as in the distance model in Equation 6), the vector model of Equation 7 presumes that the subject favors one end of each dimension and prefers those stimuli that lie farthest toward the favored end. Whereas distance models characterize subjects in terms of ideal point coordinates in the same space as the stimulus coordinates, vector models characterize subjects with weights, $w_{k s}$, constituting a space separate from the stimulus space. The sign of the weight, $w_{k s}$, indicates whether the subject favors the positive or negative end of dimension $k$, and the absolute value is said to reflect the dimension salience.

External analysis models. In external models, the stimulus coordinates are presumed known from either theory or a prior scaling. The object is to estimate subject parameters and to assess the fit of the model to the data and to known stimulus coordinates. Most external analyses employ models from Carroll's (1972) hierarchy. Only the most frequently used models will be described here.

At the lowest level of the hierarchy is the vector model in which preferences are presumed to satisfy some form of Equation 7. Methods of fitting the model yield estimates of subject salience weights, $w_{k s}$, and a measure of fit.

In the weighted Euclidean model, except for error, preferences are presumed related to the known stimulus coordinates by a function of the following form:

$\delta_{i s}=\mathrm{f}_{s}\left[\sum_{k} w_{k s}^{2}\left(\mathrm{x}_{i k}-\mathrm{y}_{s k}\right)^{2}\right]$.

This model presumes subjects to vary in the locations of their ideal points, $y_{s k}$, and in dimension saliences, $w_{k s}$. Consequently, algorithms for fitting this model yield two sets of subject parameters. In metric algorithms that use a squared error loss function, the fit is measured by a multiple correlation coefficient, the significance of which can be tested with the conventional $F$ statistic. Furthermore, if a metric model is assumed, it can be shown that the weighted Euclidean model hierarchically includes the vector model as a special case; hence, a standard $F$ statistic exists for testing whether the weighted Euclidean model accounts for signifi- cantly more variance than the vector model. Carroll and Chang's (1967) PREFMAP is the most widely used program, but several others exist: DACAR (Davison, 1976, 1980); LINMAP (Srinivasan \& Shocker, 1973); and MORALS (de Leeuw, Young, \& Takane, 1976).

Most additive numerical conjoint analyses are built on the vector model. In numerical conjoint analysis, each stimulus corresponds to one cell of an ANOVA design. For instance, the stimuli might be written descriptions of bank accounts varying on two factors: interest rate and minimum balance. Each factor might contain three levels-5\%, 7\%, and $9 \%$ for the interest factor and $\$ 100, \$ 1,000$, and $\$ 2,500$ for the minimum balance factor - to yield nine stimuli, one for each cell in the $3 \times 3$ ANOVA design. Subjects would then rate or rank the nine stimuli in terms of how much they liked each account (or how likely they would be to open such an account). In the external analysis of the data, the known stimulus coordinates, $x_{i k}$, would represent dummy codings of ANOVA effects. If $x_{i k}$ is a coordinate designating the presence $\left(x_{i k}=\right.$ 1) or absence $\left(x_{i k}=0\right)$ of feature $k$ in stimulus $i$, then the empirically estimated weight, $w_{k s}$, is usually interpreted as the utility of feature $k$ to subject $s$.

The proximity and preference models reviewed above constitute the major MDS developments leading to the statistical issues and applied studies reviewed in this special issue.

\section{Overview Or Papers}

In the first paper, Heiser and Meulman consider the application of constraints to either the parameters or the data function in MDS models. The authors discuss the role of substantive theory in MDS research, including the role theory can play in suggesting constraints. They describe the various possible kinds of constraints. Their discussion of constraints leads to a comparison of MDS with other multivariate methods. Finally, the authors consider the sampling error associated with MDS solutions and techniques for confirming (or disconfirming) structural hypotheses.

Spence reviews monte carlo simulation studies of MDS algorithms. These studies lead to several 
recommendations, including recommendations about choosing a computer program, deciding dimensionality, and dealing with large stimulus sets. Spence also discusses problems in designing good monte carlo research.

In the third paper, Cooper reviews the MDS research in consumer psychology. He covers MDS's contribution in six marketing domains: product planning, product pricing and brand naming, choosing distribution channels, personal selling, advertising, and general fact finding. He then offers three suggestions. First, marketing research should move toward a more fine-grained analysis of individual and group perceptions of products. Second, it should merge MDS data on product perceptions with other consumer level measurements (such as normative beliefs) and with market level measurements. Third, it should expand research to include, not only mew products in existing markets, but also new markets such as those created by video games and home computers.

Jones examines the literature on social perception. He divides the area into studies of interpersonal perception and attraction in intact groups, perception of political and fictional figures, and perception of social roles, relationships, and situations. He points out that the field seeks dynamic process models, whereas MDS provides static, structural representations. MDS and related techniques can yield representations of perceived social stimuli over which process models are presumed to operate.

The fifth paper, by Shoben, reviews the MDS literature in cognitive psychology. He first describes studies designed simply to represent stimulus structure; then he discusses studies of the functional relationship between MDS stimulus structure and behavioral responses, such as reaction times in categorization tasks. His third set of studies focuses on changes in structure as a function of age and context. Finally, Shoben compares MDS and cluster models as techniques for mapping cognitive structure.

In the vocational psychology literature reviewed by Rounds and Zevon, mosi studies have focused on the structure of vocational interests and on perceptions of occupations. Increasingly, however, re- searchers use MDS to study vocational development, sources of job satisfaction, and work outcomes. The authors call for more attention to the sampling of stimuli in MDS studies. The emerging confirmatory techniques offer ways to examine the field's various theories of structure.

Taken together, these six papers illustrate MDS's past and future directions. Some common themes run through the papers. First, the difficulty of handling large data sets poses an impediment to applied MDS research. Second, in applied areas, there is movement away from studies that simply describe stimulus structures toward those that explicitly examine theories of structures, that study changes in structure, or that study the relationship between perceived stimulus structure and behavioral responses to the stimuli. Third, there remain numerous questions as to the relative merits of the stimulus representations provided by factor, discriminant, MDS, and cluster analysis. The six authors describe these and other common themes far better than this brief introduction.

\section{References}

Amold, J. B. A multidimensional scaling study of semantic distance. Joumal of Experimental Pychology Monograph, 1971, 90, 349-372.

Atmeave, F. Dimensions of similarity. American Journal of Pychology, 1950, 63, 516-556.

Bloxom, B. Individual differences in multidimensional scaling (Research Bulletin 68-45). Princeton NJ: Educational Testing Service, 1968.

Carroll, J.D. Individual differences and multidimensional scaling. In R. N. Shepard, A. K. Romney, \& S. B. Nerlove (Eds.), Multidimensional scaling: Theory and applications in the behavioral sciences (Vol. 1). New York: Seminar Press, 1972.

Carroll, J. D., \& Arabie, P. Multidimensional scaling. Annual Review of Psychology, 1980, 31, 607-649.

Carroll, J. D., \& Chang, J. I. Relating preference data to multidimensional scaling via a generalization of Coombs' unfolding model. Paper presented at the annual meeting of the Psychometric Society, Madison WI, April 1967.

Carroll, $\mathbb{J}$. D., \& Chang, $J$. Analysis of individual differences in multidimensional scaling via an $N$-way generalization of "Eckart-Young" decomposition. Psychometrika, 1970, 35, 283-319.

Carroll, J. D., \& Chang, J. J. IDIOSCAL (Individual Differences In Orientation SCALing): A generaliza-

Downloaded from the Digital Conservancy at the University of Minnesota, http://purl.umn.edu/93227. May be reproduced with no cost by students and faculty for academic use. Non-academic reproduction requires payment of royalties through the Copyright Clearance Center, http://www.copyright.com/ 
tion of INDSCAL allowing idiosyncratic reference systems as well as an analytic approximation to IND. SCAL. Paper presented at the annual Psychometric Society meeting, Princeton NJ, March 1972.

Coombs, C. H. A theory of data. New York: Wiley, 1964.

Coxon, A. P, M. The user's guide to multidimensional scaling. Exeter NH: Heinemann Educational Books, 1982.

Davison, M. L. Fitting and testing Carroll's weighted unfolding model for preferences. Psychometrika, 1976 , $41,233-248$

Davison, M. L. DACAR: A FORTRAN program for fitting linear and distance models of preference. Applied Psychological Measurement, 1980, 4, 419.

Davison, M. L. Multidimensional scaling. New York: Wiley, 1983.

de Leeuw, I., Young, F. W., \& Takane, Y. Additive structure in qualitative data: An altemating least squares method with optimal scaling features. Psychometrika, $1976,41,471-503$.

Eckart, C., \& Young, G. Approximation of one matrix by another of lower rank. Psychometrika, 1936, 1, $211-218$.

Gower, J. C. Some distance properties of latent root and vector methods used in multivariate analysis. Biometrika, $1966,53,325-338$

Guttman, L. A general nonmetric technique for finding the smallest coordinate space for a configuration of points. Psychometrika, 1968, 33, 469-504.

Horan, C. B. Multidimensional scaling: Combining observations when individuals have different perceptual structures. Psychometrika, 1969, 34, 139-165.

Kruskal, J. B. Multidimensional scaling by optimizing goodness-of-fit to a nonmetric hypothesis. Psychometrika, 1964, 29, 1-28, 115-129.

Kauskal, J. B., \& Carmone, F. How to use M-D-SCAL (Verson 5M) and other useful information. Unpublished manuscript, Bell Laboratories, undated.

Kruskal, J. B., \& Wish, M. Multidimensional scaling. Beverly Hills CA: Sage Publications, 1978.

Kruskal, I. B., Young, F. W., \& Seery, J. B. How to use KYST, a very flexible program to do multidimensional scaling and unfolding. Unpublished manuscript, Bell Laboratories, 1973.

Lingoes, J. C. The Guttman-Lingoes nonmetric program series. Ann Arbor MI: Mathesis Press, 1973.

Pruzansky, S. How to use SINDSCAL: A computer program for individual differences in multidimensional scaling. Unpublished manuscript, Bell Laboratories, 1975.

Ramsay, J. O. Some statistical considerations in multidimensional scaling. Psychometrika, 1969, 34, 167181.

Ramsay, I. O. MULTISCALE: Four programs for multidimensional scaling by the method of maximum like- lihood. Chicago: International Educational Services, 1978.

Ramsay, J. O. Some small sample results for maximum likelihood estimation in multidimensional scaling. Psychometrika, 1980, 45, 139-144.

Richardson, M. W. Multidimensional psychophysics. Psychological Bulletin, 1938, 35, 659-660.

Schiffman, S. S., Reynolds, M. L., \& Young, F.W. Introduction to mulridimensional scaling. New York: Academic Press, 1981.

Schönemann, P.H. On metric multidimensional unfolding. Psychometrika, 1970, 35, 349-366.

Shepard, R. N. The analysis of proximities: Multidimensional scaling with an unknown distance function. Psychometrika, 1962, 27, 125-140, 219-246.

Shepard, R. N., \& Carroll, J. D., Paramerric representation of nonlinear data structures. In P. R. Krishnaiah (Ed.), International sympositum on mulivariate analysis. New York: Academic Press, 1966.

Srinivasan, V., \& Shocker, A. D. Linear programming techniques for multidimensional analysis of preferences. Psychometrika, 1973, 38, 337-369.

Takane, Y. Multidimensional successive categories scaling: A maximum likelihood method. Psychometrika, $1981,46,9-28$.

Takane, $Y$. \& Sergent, I. Multidimensional scaling models for reaction times and same-different judgments. Psychometrika, 1983, 48, 393-423.

Torgerson, W. S. Multidimensional scaling: I. Theory and method. Psychometrika, 1952, 17, 401-419.

Torgerson, W. Theory and methods of scaling. New York: Wiley, 1958.

Tucker, $L$. $\mathbb{R}$. Intra-individual and inter-individual multidimensionality. In H. Gulliksen \& S. Messick (Eds.), Pychological scaling: Theory and applications. New York: Wiley, 1960.

Tucker, L. R. Relations between multidimensional scaling and three-mode factor analysis. Psychometrika, 1972, 37, 3-28.

Young, F. W., \& Lewyckyj, R. ALSCAL 4 User's Guide (2nd ed.). Chapel Hill NC: Data Analysis and Theory Associates, 1979.

Young, F. W., \& Torgerson, W. S. TORSCA: A FORTRAN IV program for Shepard-Kruskal multidimensional scaling analysis. Behavioral Science, 1967, 12, 498.

Young, G., \& Householder, A. S. Discussion of a set of points in terms of their mutual distances. Psychometrika, $1938,3,19-22$.

\section{Author's Address}

Send requests for reprints or further information to Mark L. Davison, Department of Educational Psychology, University of Minnesota, 178 Pillsbury Drive S.E., Minneapolis MN 55455, U.S.A. 